\title{
ANÁLISIS DE LA INCORPORACIÓN DE LOS HOMBRES Y LAS MASCULINIDADES EN LOS PLANES ESTRATÉGICOS DE IGUALDAD EN ESPAÑA
}

\section{Resumen}

La desigualdad entre mujeres y hombres hace necesaria la acción de los poderes públicos para su erradicación. Tras 30 años de Políticas de Igualdad, son necesarias estrategias que aborden con mayor eficacia la complejidad de las relaciones de género. Las políticas de igualdad han puesto el foco en la lucha contra la discriminación de las mujeres y en el fomento de su participación en la sociedad. Sin embargo, no son muchas las medidas concretas que se han diseñado para promover el cambio en los hombres, a pesar de las recomendaciones de diferentes organismos internacionales. A través de una revisión de los planes de igualdad elaborados en España, estatales y autonómicos, analizaremos el tipo de medidas dirigidas a los hombres que se están diseñando. El análisis se enmarca en la discusión sobre cómo las políticas públicas pueden promover la transformación de los hombres y del significado de la masculinidad en nuestras sociedades.

Palabras clave: Políticas de igualdad, planes de igualdad, género, masculinidades.

\begin{abstract}
Analysis of the Inclusion of Men and Masculinities in the Strategic Plans for Equality in Spain

The inequality between women and men makes the action of the public powers necessary for its eradication. After 30 years of equality policies, strategies are needed that more effectively address the complexity of gender relations. Equality policies have focused on combating discrimination against women and promoting their participation in society. However, few concrete measures have been designed to promote change in men, despite the recommendations of different international organizations. Through a review of the equality plans drawn up in Spain, at both state and autonomous community level, we will analyze the type of measures aimed at men that are being designed. This analysis is part of the discussion on how public policies can promote the transformation of men and the meaning of masculinity in our societies.
\end{abstract}

Keywords: Equality policies, plans for equality, gender, masculinities.

Doctoranda en Trabajo Social en la Universidad Complutense de Madrid, 28223 - Pozuelo de Alarcón, Madrid, España.

Dirección postal: c/ Sigerico 5, 28019 - Madrid, España.

Correo electrónico: bakea@cepaim.org 


\begin{abstract}
Resumo
Análise da inclusão dos homens e das masculinidades nos planos estratégicos para a igualdade em Espanha

A desigualdade entre mulheres e homens torna necessária a ação dos poderes públicos para a sua erradicação. Após 30 anos de políticas de igualdade, são necessárias estratégias que abordem eficazmente a complexidade das relações de género. As políticas de igualdade têm-se focado no combate à discriminação contra as mulheres e na promoção da sua participação na sociedade. Porém, poucas medidas concretas foram concebidas para promover a mudança nos homens, apesar das recomendações de diferentes organizações internacionais. Através de uma revisão dos planos para a igualdade elaborados em Espanha, tanto a nível estatal como das comunidades autónomas, analisaremos o tipo de medidas dirigidas aos homens que estão a ser construídas. A análise enquadra-se na discussão sobre o modo como as políticas públicas podem promover a transformação dos homens e do significado da masculinidade nas nossas sociedades.
\end{abstract}

Palavras-chave: Políticas de igualdade, planos para a igualdade, género, masculinidades.

\title{
Introducción
}

La situación de las mujeres y las niñas en el mundo (ONU 2020) hace necesario que los poderes públicos desarrollen políticas que incidan en la desigualdad de género. En España, tras tres décadas de políticas de igualdad, la desigualdad entre mujeres y hombres sigue presente en muchos ámbitos. La violencia que todavía sufren las mujeres por el hecho de serlo es incompatible con la pretensión de un Estado democrático y de derecho. Sumado a esto, la pandemia de COVID-19 no sólo ha hecho más visibles estas desigualdades, sino que, en muchos casos, las ha incrementado (OIT 2020).

Si bien la Constitución española afirma la igualdad entre mujeres y hombres, también plantea la necesidad de remover los obstáculos y promover que dicha igualdad sea efectiva. Desde su aprobación en 1978, y gracias en gran parte al movimiento feminista, se trasladaron a la esfera política reivindicaciones que se tradujeron en la implementación de políticas públicas de igualdad (Roldan 2006) destinadas a lograr la corrección de las desigualdades sociales entre los sexos.

Las administraciones públicas en España cuentan con un instrumento donde se plasman los objetivos y compromisos en materia de igualdad entre mujeres y hombres: los llamados, en las primeras fases, Planes de Igualdad ${ }^{1}$ de Oportunidades para las Mujeres y, más recientemente, Planes Estratégicos de Igualdad. Dichos planes han contemplado el análisis de la situación y acciones concretas en relación con las mujeres, pero nos preguntamos cómo ha sido el tratamiento dado a los hombres y las masculinidades y sin han sido objeto de análisis y/o de medidas concretas de acuerdo con las recomendaciones de diferentes organismos internacionales.

$1 \quad$ No confundirlos con los Planes de Igualdad de las empresas que definió la Ley de Igualdad de 2007. 
Hasta fechas recientes, las políticas de igualdad han estado dirigidas a las mujeres con la intención de favorecer la igualdad de oportunidades de éstas (Astelarra 2005). Sin embargo, en su análisis de políticas públicas de España y Europa, ya Bustelo y Lombardo (2009) se preguntaban si las medidas para promover la igualdad de género llegaban a cuestionar el poder masculino y a pedir el cambio en los hombres. Las autoras concluirán que había "una tendencia a representar a las mujeres como sujetos que tienen el problema y grupo objetivo de las medidas", y constatarán "la ausencia de una llamada al cambio y a la acción para los hombres" (ibid. 17).

Sin embargo, en diferentes documentos internacionales se ha subrayado la importancia del papel que pueden jugar los hombres para la disminución de las brechas de género (ONU 2004, 2008, 2015; Ministry of Social Affairs and Health 2006; Comisión Europea 2012). Hace más de 25 años, en la Conferencia de las Mujeres de Beijing, se "alienta a los hombres a que participen plenamente en todas las acciones encaminadas a garantizar la igualdad de mujeres y hombres" (ONU 1995). En 2011 el conocido como Convenio de Estambul² señalará que las estrategias de prevención de la violencia hacia las mujeres debían incluir a los hombres y a los niños. La Estrategia Europea para la Igualdad de Género 2020-2025 (Comisión Europea 2020) indica que la prevención de la violencia centrada en hombres, niños y masculinidades debe ser de vital importancia.

En España, sin embargo, la incorporación de los hombres en las políticas públicas de igualdad apenas ha sido objeto de reflexión y práctica. Hasta la fecha, las políticas de igualdad para hombres han sido más una reivindicación del Movimiento de Hombres por la Igualdad (Sáez y Lozoya 2019) y de otras organizaciones de la sociedad civil (Alonso y López 2019) que objeto de reflexión por parte de la academia.

En este contexto nos proponemos analizar los planes de igualdad estatales y autonómicos a la luz de algunas propuestas que se han hecho a nivel internacional para que las políticas de igualdad se dirijan a los hombres, incluyendo los posibles riesgos.

\section{Método}

Para el análisis de los Planes de Igualdad, estatales y autonómicos, se ha llevado a cabo una búsqueda documental en Internet. La investigación documental de textos oficiales ha sido utilizada para el análisis de las políticas de igualdad por autoras como Verloo y Lombardo (2007), Bustelo y Lombardo (2009) y, más recientemente, Jiménez (2020). El análisis de los textos de los planes nos permite ver

Convenio del Consejo de Europa sobre prevención y lucha contra la violencia contra las mujeres y la violencia doméstica. 
cómo se problematiza la desigualdad de género, la definición de lo actores que deben intervenir y el tipo de medidas que se diseñan para cumplir con los objetivos que se marcan. Es cierto que el análisis documental se muestra insuficiente para ver todas las fases de las políticas públicas, pero se muestra eficiente para analizar la fase del diseño que aquí nos interesa.

En cuanto al acceso a las fuentes de información, los planes estatales han sido localizados en la página web del Instituto de la Mujer. ${ }^{3}$ Los planes autonómicos han sido localizados en las páginas web de los Institutos de la Mujer de las comunidades autónomas o en las propias páginas web de éstas últimas.

El análisis de los planes comprende un período que abarca desde el primer plan estatal elaborado en 1988 hasta los autonómicos más recientes. Sobre la temporalidad, existen diferencias importantes en el número y periodicidad en la elaboración de planes, por lo que tomaremos el último plan de cada una de las comunidades autónomas aprobado. Una vez localizados los 25 planes (6 estatales, 17 autonómicos y los 2 de las ciudades autónomas), se ha prestado especial atención a dos cuestiones:

- ¿Se señala en los diagnósticos u objetivos que los hombres tengan que cambiar o hacer algo para que sea posible la igualdad?

- ¿Existen medidas destinadas de forma específica a los hombres? ¿Cuáles y en qué áreas/ejes?

\section{Marco teórico}

Nos acercamos al objeto de estudio desde los estudios de masculinidades con un enfoque constructivista conectado con los discursos feministas. En concreto, los llamados "estudios críticos de la masculinidad" (Hearn et al. 2004) cuya pretensión no es sólo el conocimiento de los hombres sino su transformación. El concepto de masculinidad que se ha venido debatiendo y redefiniendo desde los años 1970 pivota sobre un término clave que popularizó Connell $(1997,39)$, la masculinidad hegemónica, entendida como "la configuración de práctica genérica que encarna la respuesta corrientemente aceptada al problema de la legitimidad del patriarcado, la que garantiza (o se toma para garantizar) la posición dominante de los hombres y la subordinación de las mujeres".

Las masculinidades son construcciones sociales que varían a lo largo del tiempo. Si bien entendemos el género como una categoría de análisis y una herramienta fundamental para entender las desigualdades y cómo se debe intervenir sobre las mismas (Scott 1990), con demasiada frecuencia el género se utiliza como sinónimo de mujer, a pesar de que la información sobre las mujeres es siempre información sobre los hombres. Sin embargo, es precisamente la construcción de

$3 \quad$ https: / / www.inmujer.gob.es / 
género de los hombres lo que hace necesario que "las políticas de igualdad empiecen a tener como destinatarios también a los hombres" (Salazar 2013, 40). Las políticas de igualdad dirigidas a los hombres estarían destinadas a desmontar los pilares de esa masculinidad hegemónica o en palabra de Bourdieu (2000), la dominación masculina sobre las mujeres.

Sucede que, para el caso de los hombres, la construcción del género camina por dos senderos paralelos. Por un lado, los hombres obtienen enormes beneficios por formar parte de un grupo social privilegiado, pero, por otro, la masculinidad hegemónica supone para éstos una menor esperanza de vida, mayores tasas de drogodependencias, fracaso escolar, accidentes de tráfico, sobrerrepresentación en las cárceles, muertes violentas, suicidios, etc. (Connell 1997).

En España hay una escasez de investigaciones sobre hombres y masculinidades, no existiendo líneas de investigación y de asignaturas específicas de masculinidades en las universidades españolas (Quiles 2019). Por el contrario, en otras latitudes hay aportaciones importantes - en el ámbito anglosajón, Bob Pease (2001) con sus reflexiones desde el trabajo social, y sobre políticas públicas y hombres, autores como Jeff Hearn (2006); en el contexto latinoamericano, encontramos referencias sobre políticas públicas, hombres y masculinidades (Alatorre 2006; Aguayo y Sadler 2011; Barker y Greene 2011; Garda 2011; Menjívar 2012; Figueroa 2014; Tena 2014; Aguayo et al. 2016; Ramírez y Gutiérrez de la Torre 2017) con una variedad de estudios de caso. En líneas generales, la literatura señala que las políticas no han entendido a los hombres como género, considerando que todos los hombres son iguales y poseen la misma cantidad de poder y asumiendo, de alguna manera, que los hombres no pueden cambiar.

Desde el análisis de las políticas de igualdad, nos situamos en la clasificación que ofreció Astelarra (2009), que distingue tres fases: actualización de toda la normativa al principio de igualdad y no discriminación; acciones positivas destinadas a contrarrestar las desventajas históricas sufridas y el mainstreaming de género, en la que se establece la necesidad de que la perspectiva de género sea transversal a todas las políticas y sectores. La incorporación del concepto de mainstreaming supone un cambio en las políticas de igualdad y ya no se trata tanto de diseñar medidas específicas para mujeres sino de introducir una mirada de género en todas las políticas.

\section{Resultados}

En el Anexo 1 hay detalle de las medidas localizadas en cada plan. Resumimos aquí los más significativos, según se desprendan de los planes estatales o autonómicos y de acuerdo a las dos preguntas de investigación relacionadas con los diagnósticos y las medidas. 


\section{Planes estatales}

$\rightarrow$ El primero (1988-1990) y el segundo (1993-1995) están diseñados como estrategia para mejorar la situación social de las mujeres. En el III plan se señala que la promoción de la igualdad no requiere únicamente la adopción de medidas positivas dirigidas a las mujeres, también requiere medidas destinadas a adaptar la organización de la sociedad hacia una distribución más justa de roles. No es hasta el IV plan (2003-2006) que los hombres son mencionados explícitamente. En el diagnóstico se señala que "sin la implicación de los hombres no habrá reparto equitativo", afirmando que la implicación de los hombres en lo doméstico es una asignatura pendiente. En el V plan (2008-2011) se marca como objetivo "fomentar el uso del permiso de paternidad y vigilar posibles discriminaciones que puedan sufrir los padres". En el diagnóstico del área de salud, se dice que "los hombres tienen mayores riesgos debido a la masculinidad".

$\rightarrow$ En el IV plan aparece como medida "estudiar la posibilidad de ampliar el permiso de paternidad y estudiar las causas por la que los hombres no hacen uso de sus derechos en la materia" y el desarrollo de campañas que promuevan la implicación de los hombres en las tareas (aunque dice dirigidas a "la población en general"). También, señala la sensibilización y cambio de actitudes, especialmente entre los hombres, y favorecer la implicación de los padres en la salud infantil. En el eje de violencia se busca la extensión de programas específicos de reeducación y tratamiento psicológico dirigido a las personas ${ }^{4}$ condenadas por violencia de género. En el V plan (2008-2011) aparecen nuevos indicadores como "la tasa de actividad doméstica masculina". En el último plan aprobado (2014-2016) hay una medida para promover la implicación de los hombres en las tareas del cuidado y el desarrollo del vínculo afectivo y de apego, así como actuaciones de sensibilización y difusión de la parentalidad positiva.

\section{Planes autonómicos}

$\rightarrow$ En cuanto a los objetivos, 6 planes se marcan el trabajo con hombres como estrategia para el logro de la igualdad (Aragón, Castilla y León, Castilla y La Mancha, Extremadura, Galicia y La Rioja). Los planes de algunas Comunidades Autónomas van incluso más allá al incluir en las presentaciones referencias explícitas a los hombres. El plan de la Rioja señala que "es objetivo el cambio de valores especialmente en los hombres (...)". En la presentación del plan de Castilla y La Mancha se menciona como una

Sorprende el uso del término "persona", aunque la Ley contra la Violencia de Género de 2004 señala a los hombres como los perpetradores de este tipo de violencia. 
de las novedades del plan las "nuevas masculinidades" y se habla de "modelo masculino hegemónico". En el plan de Asturias, en la introducción, se señala que "la igualdad con respecto al género no es propiamente un problema de las mujeres, concierne también a los hombres".

$\rightarrow$ A pesar de que no todos los planes tienen a los hombres en sus objetivos explícitos, la mayoría incluyen medidas dirigidas, específicamente, a éstos. Las áreas donde aparecen medidas para hombres son los de violencia y conciliación/corresponsabilidad. La medida prioritaria gira en torno al permiso de paternidad. En ocasiones, el trabajo con hombres se plantea sólo con algunos colectivos, como el caso de "hombres reclusos" (plan de Murcia). Los planes de La Rioja y Extremadura mencionan a los grupos de hombres. El primero marca como medida "fortalecer la participación ciudadana de asociaciones de mujeres, de movimientos feministas y de grupos de hombres por la Igualdad". El segundo se marca como objetivo "impulsar la creación de asociaciones o grupos de reflexión de hombres en relación con las masculinidades". El plan de Castilla y León propone la participación de las asociaciones de hombres por la igualdad y propiciar encuentros entre las asociaciones de mujeres y las asociaciones de hombres pro-igualdad. El plan del País Vasco se destaca en la incorporación de medidas así como en el diseño de indicadores, siendo además el único que menciona un programa específico, Gizonduz.

\section{Discusión}

Los hombres siempre han sido objetivo de las políticas públicas. Lo novedoso, en nuestro objeto de estudio, radica en analizar si se actúa con ellos en cuanto hombre, como seres que participan en las relaciones de género (Flood 2015).

Para nuestro análisis son especialmente interesantes los trabajos de Ramírez y De la Torre (2017) en México sobre la construcción de una agenda de políticas públicas de género de los hombres donde se identifican los temas-problemas, las instancias que podrían ofrecer soluciones, actores involucrados, alianzas necesarias, así como las propuestas de políticas públicas. El esquema seguido podría servir de base para recorrer un camino parecido en España, dado que lo que muestra nuestro análisis de los planes es que no hay una estrategia común y que cada comunidad autónoma ha ido incorporando el asunto de los hombres de manera diferente. En lo único en lo que todos los planes coinciden es en enmarcar las medidas dirigidas a los hombres (cuando lo hacen) en los ejes de violencia y conciliación/corresponsabilidad, a pesar de que los estudios y documentos internacionales plantean ir bastante más allá, como veremos.

Barker y Greene (2011) plantearon cuatro principios para la inclusión de los hombres en las políticas públicas: la necesidad de asegurar derechos, que las polí- 
ticas estén basadas en evidencia, que partan de una necesaria mirada relacional de género y que se articule la igualdad de género con la pobreza y la exclusión social. Con excepción de la medida estrella del permiso de paternidad, ninguno de los principios se estaría cumpliendo en los planes autonómicos y estatales analizados.

Por su parte, Connell (2003) realizó recomendaciones a los poderes públicos que podrían orientar el diseño de políticas de igualdad y que, de momento, no se ven plasmado mayoritariamente en los planes analizados:

- nombrar a los hombres y a los niños como agentes de cambio

- reconocer la capacidad de hombres y niños para cambiar las relaciones en las que están envueltos y su responsabilidad

- conocer las diferencias y diversidades entre los propios hombres y sus intereses

Además de las consideraciones de los expertos, hay que subrayar que desde el movimiento feminista hay recelo a que se inviertan fondos públicos y que los escasos recursos disponibles que hay para las mujeres se dediquen también para trabajar con los hombres. Garda (2011) alertó, además, de que algunos enfoques podrían ser erróneos a la hora de incluir a los hombres. En ocasiones, parece que se pretende compartir responsabilidades en las problemáticas de género entre mujeres y hombres. También alerta acerca de que las políticas para las mujeres desaparecen como tales. Le preocupa la ausencia de un sujeto político que demande éstas políticas, y sí estas políticas deben incluir en sus costos la generación de consciencia para quien va dirigida. Este último punto nos alertaría de las medidas de los planes analizados que incluyen campañas de sensibilización hacia los hombres.

\section{Sobre los diagnósticos}

Las políticas públicas se diseñan a partir de diagnósticos de la realidad social. Si el significado de ser hombre - construido culturalmente - está asociado mayoritariamente a roles y expectativas que, a la larga, desembocan en actitudes y practicas violentas y machistas (Lorente 2009), ¿no deberían las políticas de igualdad dirigirse a revertir dicha construcción?; ¿son suficientes las medidas de "empoderamiento" femenino o de promoción de las mujeres? De los planes analizados son pocos los que en los diagnósticos hacen alguna reflexión sobre qué les pasa a los hombres más allá de presentar una batería de estadísticas que comparan la situación de mujeres y hombres en diferentes esferas.

Nos preguntamos por qué el tema no ha entrado en la agenda pública española sobre igualdad. Un hecho determinado (la relación entre masculinidad y violencia, por ejemplo) se convierte en parte de la agenda pública si se dan tres condiciones: si se convierte en tema, en un problema público y si se dota de carga 
política (PNUD 2008). Por tanto, problematizar la masculinidad y a los hombres es el primer paso necesario para que se puedan diseñar políticas públicas al respecto. Cabe preguntarse por qué unos problemas llegan a formar parte del programa de acción de los poderes públicos y otros no. En la literatura sobre políticas públicas se señala que los temas entran en agenda, fundamentalmente, por dos vías: interna (partidos políticos, organismos estatales, etc.) y externa (sociedad civil) (Subirats 1989).

En relación al papel de los partidos políticos en España, las mujeres feministas han impulsado fuertemente el desarrollo de políticas de igualdad y de un feminismo institucional en las últimas décadas. Sin embargo, sobre la posibilidad de dirigir políticas de manera específica a los hombres no hay una posición clara. Al respecto, el contrapunto viene desde la extrema derecha, que podría estar canalizando lo que se ha dado en llamar "crisis de la masculinidad" (Kaufman 1993). Vox, en sintonía con las proclamas de los movimientos por los derechos de los hombres, se ha alineado con corrientes anti-gender (Kuhar y Paternotte 2017) presentando a los hombres como víctimas de las políticas de igualdad.

En cuanto al papel del movimiento feminista, no existe en España una corriente de opinión que esté abogando mayoritariamente por la inclusión de los hombres en las políticas de igualdad. De todas formas, se observan algunos cambios: los debates sobre prostitución comienzan a centrarse cada vez más en los "puteros" que en las mujeres prostitutas (Ranea 2019). Ana de Miguel (2016) dedica las últimas páginas de su Neoliberalismo Sexual a los hombres. El problema del consumo masivo de pornografía y los problemas asociados con el mismo no puede entenderse sin una reflexión sobre la construcción de la sexualidad masculina y su relación con el poder (Cobo 2020).

Otra de las cuestiones que se observa en los planes analizados es que género se usa como sinónimo de mujer, a pesar de que la perspectiva de género "permite analizar las posibilidades vitales de unas y otros, el sentido de sus vidas, sus expectativas y oportunidades y las complejas y diversas relaciones que se dan entre los sexos"' (Lagarde 1996).

\section{Medidas y áreas de intervención}

Hay autoras que ya han señalado que ha faltado una actuación explícita a favor de la igualdad por parte de otras políticas públicas que aunque no podamos catalogar como de igualdad sí que afecta a las posibilidades reales de una igualdad efectiva (Lombardo y León 2014). Si bien la mayoría de los planes analizados citan el mainstreaming como estrategia, no se observa una mirada de género - que además sea sobre mujeres y hombres - en la batería de medidas que despliegan. Esto no sucede sólo en el caso español; aún cuando la definición de mainstreaming de género incluye a los hombres, en la mayoría de países los hombres no han sido un objetivo como estrategia para la igualdad de género (Scambor y Scambor 2009). 
Para la inclusión de los hombres en las políticas de igualdad, el documento "Men and Gender Equality Policy in Finland" (apud Abril 2010) señala dos estrategias: acciones que apoyan el rol de los hombres a la hora de mejorar la situación de las mujeres (ej: corresponsabilidad en los cuidados), y acciones que reduzcan los problemas que afectan específicamente a los hombres (ej: accidentes de tráfico). Las medidas que incluyen los planes analizados estarían situadas en el primer tipo de acciones, con excepción de las problemáticas relacionadas con la salud ${ }^{5}$ masculina.

De cara a ver si las medidas que se plasman en los planes en España recogen las que se recomienda en la literatura internacional, nos remitimos a la revisión que llevan a cabo ICRW y Promundo (2010) sobre diferentes políticas dirigidas a hombres. Sugieren que las políticas que pretendan incluir a los hombres deben: incorporar el área de educación y especialmente en la infancia; políticas de seguridad pública, que engloban a las fuerzas armadas, policía y prisiones; políticas dirigidas a grupos específicos de hombres con mayor vulnerabilidad; programas sobre salud masculina que incluya sexualidad y salud reproductiva; violencia de género; políticas contra la pobreza; y programas sobre paternidades positivas y cuidados.

La mayoría de estas propuestas no están recogidas en los planes analizados. Con respecto a las medidas de prevención de violencia de género (eje en el que todos los planes coinciden en incluir a los hombres), y según el documento promovido por la ONU (Aguayo et al. 2016), éstas deberían: realizar más investigaciones sobre los hombres; incorporar la prevención con hombres en las leyes y planes; elaborar campañas dirigidas a ellos; evaluar los programas dirigidos a los maltratadores; implementar programas e intervenciones con los hombres; aumentar el número de hombres como agentes de cambio y líderes contra la violencia, incorporando orientaciones basadas en evidencias. En los planes analizados lo que más se echa en falta es la promoción de investigaciones, los programas de intervención con hombres y la evaluación de los impactos en la vida de las mujeres de lo que se hace con los hombres.

En cuanto al trabajo con grupos específicos de hombres, se ha encontrado que esto puede provocar que se problematice sólo el comportamiento de algunos hombres (reclusos, por ejemplo) como si con el resto de los hombres no fuera necesario hacer nada. Y es que, si nos fijamos en las medidas de los planes analizados, los hombres son vistos como padres o como maltratadores, de facto o en potencia. El resto de hombres parecen no existir en cuanto a destinatarios de posibles medidas.

Es en el ámbito de la salud donde se han desarrollado las iniciativas más ambiciosas en otros lugares del mundo. El caso más emblemático es el de Brasil, que puso en marcha en 2009 uno de los programas más importantes en el mundo sobre la salud masculina, impulsado por el Ministerio de Salud. 
De todas formas y tal y como señaló Connell (2003), es necesario que las medidas dirigidas a los hombres hablen en concreto y positivamente acerca de ellos, sus intereses, realidades, esperanzas y problemas. Si desarrollamos medidas para los hombres, éstas deberían ser compatibles con alguno de su "intereses". Nos preguntamos si las medidas que enumeran los planes analizados se corresponden con los "intereses" de los hombres, pregunta que nos remite directamente a otra: ¿Sabemos cuáles son los intereses de los hombres? Y esta cuestión nos lleva a otro debate: ¿Pueden los hombres tener intereses no patriarcales? (Pease 2015), cuestión que sería necesario abordar para un futuro diseño de políticas dirigidas a hombres desde un enfoque de género.

Es significativo que en alguno de los diagnósticos que incluyen los planes analizados se mencionen los problemas específicos de salud de los hombres, pero no se diseña ninguna medida para intervenir sobre esta situación. Cuando el plan de Canarias señala que "los hombres, debido a sus roles de género, no reconocen su malestar físico, no sienten la necesidad de cuidarse, no hablan de sus problemas de salud ni siquiera con otros hombres y suelen acudir tarde a los servicios sanitarios", no se propone ninguna medida para atajarlo. He aquí uno de los ámbitos donde podemos ver cómo confluye lo que anteriormente señalábamos acerca de la relación entre los costes y los beneficios de la masculinidad. Por un lado, en los textos se reconoce que determinada forma de ser hombre es perjudicial para la salud de esto, pero esto no se pone en relación con lo que le pasa a las mujeres. En definitiva, no se vinculan los "costes de la masculinidad" con los "privilegios" que siguen ostentando los hombres como grupo social.

En líneas generales, las investigaciones señalan que hay que enfocar más las medidas en las estructuras que sostienen el patriarcado y menos en el cambio individual (Greig y Flood 2020) dado que existe un riesgo político de un proyecto individualizado de reforma de la masculinidad que ayudará a modernizar el patriarcado en lugar de abolirlo (Connell 2003).

Las propuestas de trabajo con hombres en España estarían llegando, sobre todo, desde los ámbitos de la intervención social que comienzan a ver indispensable trabajar con los hombres (Alonso, Aranguren y Bacete 2019). La escasa y incipiente reflexión académica no ha impedido el desarrollo de algunos programas o servicios específicos dirigidos a hombres para la transformación de las masculinidades desde administraciones públicas. Ejemplos destacados son el pionero programa de salud del Ayuntamiento de Jerez ${ }^{6}$, el programa Gizonduz ${ }^{7}$ de Emakunde, el teléfono de atención a hombres del Ministerio de Igualdad en $2009^{8}$ y el Servicio de Atención a hombres del Ayuntamiento de Barcelona9.

\footnotetext{
https: / / www.jerez.es/nc/webs_municipales/hombresxigualdad/ https://www.emakunde.euskadi.eus/gizoncas/-/informacion/gizonduz-informacion/\#QUE https: / / elpais.com/diario/2010/12/17/sociedad/1292540405_850215.html https:/ / seuelectronica.ajuntament.barcelona.cat/ oficinavirtual/es/tramit/20100000461
} 
La experiencia y evaluación de todos estos programas debería guiarnos, y que no suceda lo que indica Aguayo (2011): "hay un abismo entre las políticas esbozadas en las leyes, planes (...) y lo que sucede a nivel de la implementación de servicios públicos o financiados con recursos públicos". Es necesario dotar de conocimiento técnico y estratégico o sucederá que las acciones dirigidas a los hombres no tendrán un enfoque feminista. Idoia Eizmendi señala que hay que tener cuidado con algunas propuestas que, "en vez de acercar a los hombres a asumir responsabilidades, les llevaba a un camino victimista" (Azpiazu 2017).

\section{Conclusiones}

Podemos afirmar que los planes, tímidamente, comienzan a contemplar a los hombres como objetivo de medidas. Empieza a considerarse que, sin un cambio en los hombres, no será posible alcanzar la igualdad de género. Hasta la fecha, las medidas que se han planteado en los planes se localizan en las áreas de prevención de la violencia y de fomento de la corresponsabilidad. Sin embargo, y de acuerdo con la literatura internacional, esto resulta insuficiente. Según los principios del mainstreaming de género, las medidas dirigidas tanto a mujeres como a hombres deberían considerar otros muchos ámbitos de la vida.

En los planes analizados se observa la ausencia de un discurso que ponga en relación los costes de la masculinidad para los hombres, con sus privilegios como grupo social. No hay nomenclatura común ni líneas estratégicas marco que puedan guiar las políticas públicas que aborden el cambio social que una sociedad compleja como la del siglo XXI requiere.

A pesar de todo, algo se mueve y los hombres comienzan a entrar en la agenda de las políticas de igualdad: La Rioja ${ }^{10}$ ha iniciado diagnósticos que darán pie a programas dirigidos a los hombres. El Instituto Canario de la Mujer organizaba en 2019 el II Encuentro de Masculinidades Igualitarias ${ }^{11}$ y anunciaba la elaboración de un programa específico para el fomento de masculinidades igualitarias. La ley andaluza contra la violencia de género introdujo en 2018 una modificación para incluir actividades con hombres ${ }^{12}$. La Universidad Miguel Hernández de Elche ha puesto en marcha el primer curso de Especialista Universitario sobre Masculinidades ${ }^{13}$ y la Universidad Complutense de Madrid y la Universidad del País Vasco pronto seguirán sus pasos con un Experto sobre Masculinidades y

\footnotetext{
10 Más información en: https://www.larioja.org/igualdad/es/educacion-investigacion/diagnosticomasculinidades-igualitarias-comunidad-autonoma https:/ / www3.gobiernodecanarias.org/noticias/el-ici-celebra-en-la-laguna-el-ii-encuentro-demasculinidades-igualitarias-en-canarias/

12 Consulta BOE en: https:/ / www.juntadeandalucia.es/boja/2018/148/1

13 Más información en: http:/ / especialistamasculinidades.edu.umh.es/
} 
Acción Social. La Delegación del Gobierno contra la Violencia de Género dedicó uno de sus seminarios ${ }^{14}$, con motivos del $25 \mathrm{~N}$, a las masculinidades. La nueva Estrategia Nacional contra las Violencias Machistas recoge una línea sobre masculinidades (Reguero 2020). En noviembre de 2020 se presentaba en Sevilla el Servicio para Atención a Hombres ${ }^{15}$ impulsado por la Fundación Iniciativa Social y apoyado por el Ayuntamiento de dicha ciudad.

Es deseable que todas estas iniciativas se traduzcan en programas concretos que respondan a diagnósticos y que incluyan medidas para un cambio en los hombres, desde un enfoque feminista, sin olvidar que, fuera de España, existe ya una importante producción académica y experiencia práctica que puede ayudar en el proceso de incorporación de los hombres en las políticas de igualdad.

De cara al futuro, es necesario el diseño de nuevos indicadores que puedan dar cuenta del cambio en los hombres, el papel que pueden jugar las asociaciones de hombres por la igualdad y la sociedad civil en el diseño de una nueva generación de políticas de igualdad. El análisis de los planes contra la violencia de género, de las leyes de igualdad autonómicas, los planes de igualdad en las empresas y la evaluación del impacto en las mujeres de las medidas dirigidas a los hombres son campos de análisis que se abren. Una de las grandes cuestiones a explorar es cómo pueden las políticas públicas atajar la doble realidad de los hombres (costes y beneficios de la masculinidad), preguntándonos si las vulnerabilidades específicas de los hombres deberían ser objeto de las políticas de igualdad.

\section{Referencias}

Abril, Paco. 2010. “¿Por qué son necesarias las políticas de igualdad de género dirigidas a hombres?" Dossier Desigualtat i génere, Barcelona Societat 17: 152-161.

Aguayo, Francisco, y Michelle Sadler (eds.). 2011. Masculinidades y Políticas Públicas: Involucrando Hombres en la Equidad de Género. Santiago: Universidad de Chile.

Aguayo, Francisco, Eduardo Kimelman, Pamela Saavedra, y Jane Kato-Wallace. 2016. Hacia la incorporación de los hombres en las políticas públicas de prevención contra las mujeres y las niñas. Santiago: EME/CulturaSalud. Washington, D.C.: Promundo-US. Ciudad de Panamá: ONU Mujeres y UNFPA.

Alatorre, Javier. 2006. "Masculinidad y políticas públicas." En Debates sobre masculinidades: poder, desarrollo, políticas públicas y ciudadanía, editado por Gloria Careaga y Salvador Cruz Sierra, 303-314. Ciudad Universitaria: Universidad Nacional Autónoma de México.

Alonso, Bakea, Edurne Aranguren, y Ritxar Bacete. 2019. “El trabajo con hombres desde una perspectiva de género: una asignatura pendiente en intervención social." Zerbitzuan 69: 23-38. DOI: https: / / doi.org/10.5569/1134-7147.69.02

\footnotetext{
14 Más información sobre los seminarios: https:/ / seminariosnosqueremosvivas.es/project/ seminario2-masculinidades-machismo-es-violencia-sobre-masculinidades-y-otras-formas-de-combatirdesde-la-raiz/ 
Alonso, Bakea, y Angela López. 2019. Vistete despacio que tenemos prisa. Claves para el trabajo con hombres y masculinidades como estrategia para el logro de la Igualdad. Beniaján, Murcia: Fundación Cepaim. Disponible en: http:/ / cepaim.org/th_gallery/vistete-despacioque-tenemos-prisa/

Astelarra, Judith. 2009. Veinte años de Políticas de Igualdad. Colección Feminismos. Madrid: Cátedra.

Azpiazu, Jokin. 2017. Masculinidades y feminismo. Barcelona: Virus Editorial.

Barker, Gary, y Michelle Greene. 2011. “¿Qué tienen que ver los hombres con esto?: Reflexiones sobre la inclusión de los hombres y las masculinidades en las políticas públicas para promover la equidad de género." En Masculinidades y Políticas Públicas: Involucrando hombres en la equidad de género, editado por Francisco Aguayo y Michelle Sadler, 23-49. Santiago: Universidad de Chile.

Bordieu, Pierre. 2000. La dominación masculina. Barcelona: Anagrama

Bustelo, María, y Emanuela Lombardo. 2009. Políticas de Igualdad en España y Europa. Colección Feminismos. Madrid: Cátedra.

Cobo, Rosa. 2020. Pornografía. El placer del poder. S/1: Ediciones B.

Connell, Robert. 1997. "La organización social de la masculinidad." En Masculinidad/es. Poder y crisis, editado por Teresa Valdés y José Olavarría, 31-48. Santiago, Chile: ISIS Internacional, Ediciones de las Mujeres n. ${ }^{\circ} 24$.

Connell, Robert. 2003. "The Role of Men and Boys in Achieving Gender Equality." United Nations Division for the Advancement of Women (DAW), Expert Group Meeting, Brasilia, Brazil, 21-24 October 2003 (EGM/Men-Boys-GE/2003/ BP.1).

Comisión Europea. 2012. The Role of Men in Gender Equality - European strategies \& insights. Disponible en https: / / op.europa.eu/en/publication-detail/-/ publication / f6f90d59ac4f-442f-be9b-32c3bd36eaf1

Comisión Europea. 2020. “Una Unión de la igualdad: Estrategia para la Igualdad de Género 2020-2025." Comunicación de la Comisión al Parlamento Europeo, al Consejo, al Comité Económico y Social Europeo y al Comité de las Regiones. Disponible en https: / / eur-lex. europa.eu/legal-content/ES/TXT/HTML/ ?uri=CELEX:52020DC0152\&from=ES

De Miguel, Ana. 2016. Neoliberalismo sexual. Colección Feminismos. Madrid: Cátedra.

Figueroa, Juan Guillermo. 2014. Políticas públicas y la experiencia de ser hombre: paternidad, espacios laborales, salud y educación. México: El Colegio de México.

Flood, Michael. 2015. "Men and Gender Equality." En Engaging Men in Building Gender Equality, editado por Michael Flood y Richard Howson. Cambridge Scholars Press.

Garda, Roberto. 2011. “¿Incluir a los hombres en las políticas públicas con perspectivas de género? Por una propuesta menos liberal y más feminista y radical." Comunicación presentada en el Congreso Iberoamericano de Masculinidades y Equidad: Investigación y Activismo, Barcelona, 7-8 de Octubre.

Greig, Alan, y Michael Flood. 2020. Work with men and boys for gender equality: A review of field formation, the evidence base and future directions. Discussion Paper. New York: UN WOMEN.

Hearn, Jeff et al. 2004. The European Research Network on Men in Europe: The Social Problem and Societal Problematisation of Men and Masculinities. Vol.s 1 and 2. Brussels: European Commission.

Hearn, Jeff. 2006. "Men and Gender Equality Policy." Finnish Presidency Conference on Men and Gender Equality. Towards Progressive Policies. Helsinki, 5-6 October. Disponible en http: / / citeseerx.ist.psu.edu/ viewdoc/ summary?doi=10.1.1.631.582\&rank=1\&q=Men $\% 20$ and $\% 20$ Gender $\% 20$ equality $\% 20$ policy\&osm=\&ossid= [Acceso el 12 mayo,2021]. 
ICRW/Promundo. 2010. What men have to do with it. Public policies to promote gender equality. Disponible en https: / / promundoglobal.org/resources/what-men-have-to-do-withit-public-policies-to-promote-gender-equality / [Acceso el 13 enero, 2020].

Jiménez, Ma Luisa. 2020. "Posibilidades de la investigación documental para el análisis interseccional de las políticas de igualdad." Investigaciones Feministas 11(2): 319-331. DOI: https:/ / doi.org/10.5209/infe.66080

Kaufman, Michael. 1993. Cracking the Armour: Power, Pain and the Lives of Men. Toronto: Viking.

Kuhar, Roman, y David Paternotte. 2017. Anti-Gender Campaigns in Europe. Mobilizing against Equality. London/New York: Rowman \& Littlefield Publishers.

Lagarde, Marcela. 1996. "Nociones y definiciones básicas de la perspectiva de género." En Estudios Básicos de Derechos Humanos, compilado por Laura Guzmán y Gilda Pacheco. San José: Instituto Interamericano de Derechos Humanos.

Lombardo, Emanuela, y Margarita León. 2014. "Políticas de igualdad de género y sociales en España: origen, desarrollo y desmantelamiento en un contexto de crisis económica." Investigaciones Feministas 5: 13-35. DOI: https://doi.org/10.5209/rev_INFE. 2014.v5.47986

Lorente, Miguel. 2009. Los nuevos hombres nuevos. Colección Imago Mundi. Barcelona: Ediciones Destino.

Menjivar, Mauricio. 2012. ¿Hacia masculinidades tránsfugas? Políticas públicas y experiencias de trabajo sobre masculinidad en Iberoamérica. San José, Costa Rica: FLACSO.

Ministry of Social Affairs and Health, Finland. 2006. Men and Gender Equality. Towards Progressive Policies - Conference Report. Edited by Jouni Varanka, Antti Närhinen and Reetta Siukola. Helsinki: Ministry of Social Affairs and Health. Disponible en https:/ / julkaisut.valtioneuvosto.fi / handle/10024/74753

OIT. 2020. "Strengthening Gender Measures and Data in the COVID-19 Era: An urgent need for change." Disponible en https: / / www.ilo.org/stat/Publications/WCMS_774261/ lang--en/index.htm [Accesado 8 marzo de 2021].

ONU. 1995. Declaración y plataforma de acción de Beijing. UN Women. Disponible en https:/ / beijing20.unwomen.org/en [Acceso el 12 enero, 2021].

ONU. 2004. "The role of men and boys in achieving gender equality." Panel II. Commission on the Status of Women, $48^{\text {th }}$ session, New York. Disponible en https: / / www.un.org/ womenwatch/daw/csw/csw48/Thematic1.html [Acceso el 12 enero, 2021].

ONU. 2008. El papel de los hombres y los niños en el logro de la igualdad de género. Nueva York: ONU, División para el Adelanto de da Mujer. Disponible en https:/ / www.un.org/ womenwatch/daw/csw/csw48/Thematic1.html [Acceso el 12 enero, 2021].

ONU. 2015. Hombres, masculinidades y cambio en el poder. Un documento de debate sobre la participación de los hombres en la igualdad de género desde Beijing 1995 hasta el año 2015. Disponible en http: / / menengage.org/ wp-content/ uploads / 2014/11/Beijing-20-HombresMasculinidades-y-Cambios-en-el-Poder-MenEngage-2014.pdf [Acceso el 12 enero 2021].

ONU. 2020. El mundo para las mujeres y las niñas. Informe anual 2019-2020. ONU Mujeres. Disponible en https: / / issuu.com/carlotta.aiello/docs/es_unw_annual_report_2019-2020

Pease, Bob. 2001. "Developing profeminist practice with men in social work." Critical Social Work, 2(1): 1-8.

Pease, Bob. 2015. “¿La reconstrucción de la masculinidad o el fin de la hombría? Posibilidades y limitaciones de transformar las subjetividades masculinas para conseguir la igualdad de género." En Masculinidades alternativas en el mundo de hoy, editado por Angels Carabí y Josep Armengol, 25-46. Barcelona: Icaria. 
PNUD. 2008. Políticas de igualdad, equidad y gender mainstreaming. ¿De qué estamos hablando. Disponible en http:/ / www.americalatinagenera.org/es/documentos/doc_732_Politicas deigualdad23junio08.pdf [Acceso el 08 marzo, 2021].

Quiles, María. 2019. "Políticas de formación e investigación en género en la universidad española: estudios de masculinidad." En Masculinidades igualitarias y alternativas. Procesos, avances y reacciones, editado por Anastasia Téllez, Javier E. Martínez y Joan Sanfélix, 299-322. Valencia: Tirant lo Blanch.

Ranea, Beatriz. 2019. "Masculinidad hegemónica y prostitución femenina: (re) construcciones del orden de género en los espacios de prostitución en el estado español." Tesis, Universidad Complutense de Madrid. [https: / / dialnet.unirioja.es/ servlet/tesis?codigo=248857]

Ramírez, Juan Carlos, y Norma Gutiérrez de la Torre. 2017. Hombres y Políticas de Igualdad de Género: Una Agenda en Construcción. Guadalajara: Universidad de Guadalajara.

Reguero, Patricia (2020). "La estrategia de Igualdad contra las violencias machistas incluirá una línea de trabajo orientada solo a hombres." EL SALTO, 23 Octubre [Acceso el 01 diciembre, 2020].

Roldán, Elena. 2006. "Servicios sociales y políticas de igualdad de oportunidades." Acciones e Investigaciones Sociales 1 Ext.

Saez, Hilario, y Angel Lozoya. 2019. "Políticas públicas de Igualdad específicas para hombres." Dossier GALDE 25. Disponible en https://www.galde.eu/es/politicas-publicas-deigualdad-especificas /

Salazar, Octavio. 2013. Masculinidades y Ciudadanía. Los hombres también tenemos género. Madrid: Dykinson.

Scambor, Christian, y Elli Scambor. 2009. "Men and Gender Mainstreaming: Prospects and Pitfalls of a European Strategy." The Journal of Men Studies 16(3): 301-315. DOI: https: / / doi.org/10.3149/jms.1603.301

Scott, Joan. 2002. "El género: una categoría útil para el análisis." Op. Cit. Revista del Centro de Investigaciones Históricas 14: 9-45. Disponible en https: / / revistas.upr.edu/index.php/ opcit/article/view/16994 [Acceso el 29 abril, 2020].

Subirats, Joan. 1989. Análisis de políticas públicas y eficacia de la administración. Madrid: Ministerio para las Administraciones Públicas.

Tena, Olivia. 2014. "Incorporación del trabajo con hombres en la agenda feminista." En Debates y reflexiones en torno a las masculinidades. Analizando los caminos hacia la igualdad de género, editado por Tania Rocha y Ignacio Lozano. México: UNAM, Facultad de Psicología.

Verloo, Mieke, y Emanuela Lombardo. 2007. “Contested Gender Equality and Policy Variety in Europe: Introducing a Critical Frame Analysis Approach." En Multiple Meanings of Gender Equality. A Critical Frame Analysis of Gender Policies in Europe, editado por Mieke Verloo, 21-51. Budapest: Central European University Press. 


\section{Anexo I: Resultados}

Tabla I:

Planes estatales ${ }^{16}$

\begin{tabular}{ll}
\hline \multicolumn{1}{c}{ PLAN $^{1}$} & \multicolumn{1}{c}{ MEDIDAS } \\
\hline $\begin{array}{l}\text { IV Plan de igualdad } \\
\text { de oportunidades } \\
\text { para las mujeres } \\
(2003-2006)\end{array}$ & $\begin{array}{l}\text { Estudiar la ampliación del permiso de paternidad. Estudiar las causas } \\
\text { por la que los hombres no hacen usos de sus derechos en la materia. } \\
\text { Campañas que promuevan la implicación de los hombres en las tareas }\end{array}$ \\
\hline $\begin{array}{l}\text { V Plan Estratégico } \\
(2008-2011)\end{array}$ & $\begin{array}{l}\text { Favorecer implicación de los padres en la salud infantil. VIOLENCIA: } \\
\text { extensión de programas específicos de reeducación y tratamiento } \\
\text { psicológico dirigido a las personas condenadas por violencia de género } \\
\text { Nuevo indicador: tasa de actividad doméstica masculina }\end{array}$ \\
\hline $\begin{array}{l}\text { VI Plan estratégico } \\
(2014-2016)\end{array}$ & $\begin{array}{l}\text { Implicación hombres en las tareas del cuidado y desarrollo del vínculo } \\
\text { afectivo y apego } \\
\text { Especial atención a los permisos parentales } \\
\text { Actuaciones de sensibilización y difusión de la parentalidad positiva. }\end{array}$ \\
\hline
\end{tabular}

Elaboración propia

Tabla II:

Planes Autonómicos

\begin{tabular}{|c|c|}
\hline PLAN & MEDIDAS \\
\hline $\begin{array}{l}\text { Plan Estratégico } \\
(2010-2013) \\
\text { ANDALUCIA }^{2}\end{array}$ & $\begin{array}{l}\text { Imprescindible implicar a los hombres y a las mujeres } \\
\text { SALUD: "los hombres, desde los modelos tradicionales de masculinidad, } \\
\text { asumen mayores situaciones de riesgo aunque por otra parte también } \\
\text { duermen más horas y practican más deporte que las mujeres" } \\
\text { IMAGEN Y MEDIOS: Impulso a la generación de una imagen del hombre } \\
\text { alternativa a la del modelo tradicional machista, hombres sensibilizados en } \\
\text { materia de igualdad y que se posicionan en contra de la violencia de género }\end{array}$ \\
\hline $\begin{array}{l}\text { I Plan estratégico } \\
(2017-2020) \\
\text { ARAGÓN }\end{array}$ & $\begin{array}{l}\text { Promover campañas, incluyendo las realidades existentes donde tenga cabida } \\
\text { las nuevas feminidades y masculinidades. } \\
\text { Se incentivará el uso de medidas de conciliación por parte de los hombres }\end{array}$ \\
\hline $\begin{array}{l}\text { II Plan estratégico } \\
(2016-2019) \\
\text { ASTURIAS }\end{array}$ & $\begin{array}{l}\text { Señala: "La igualdad entre mujeres y hombres se refiere a una igualdad de } \\
\text { derechos, responsabilidades y oportunidades para las mujeres y los hombres, } \\
\text { las niñas y los niños. Esta igualdad con respecto al género no es propiamente } \\
\text { un problema de las mujeres, concierne también a los hombres" }\end{array}$ \\
\hline
\end{tabular}

16 Se han omitido de la tabla los 3 primeros planes estatales ya que ningún contempla medidas específicas dirigidas a los hombres. 


\begin{tabular}{|c|c|}
\hline PLAN & MEDIDAS \\
\hline $\begin{array}{l}\text { IV Pla Estratègic } \\
(2015-2020) \\
\text { BALEARES }\end{array}$ & $\begin{array}{l}\text { EDUCACIÓ I FORMACIÓ } \\
\text { Introduir la perspectiva de gènere en l'orientació educativa, per tal } \\
\text { d'eliminar el biaix de gènere que tendeix a orientar els homes cap a } \\
\text { àmbits com el científic i tecnològic } \\
\text { CORESPONSABILITAT I CONCILIACIÓ } \\
\text { Potenciar, des de les polítiques de sanitat, a través del personal sanitari i } \\
\text { auxiliar, la promoció de més implicació dels homes en les tasques de cura i el } \\
\text { vincle afectiu, fomentant més participació en la maternitat/paternitat i criança }\end{array}$ \\
\hline $\begin{array}{l}\text { Plan estratégico } \\
\text { 2019-2022 } \\
\text { CATALUÑA }\end{array}$ & $\begin{array}{l}\text { COEDUCACIÓN. } \\
\text { Impuls de les actuacions formatives en igualtat de gènere, noves masculinitats } \\
\text { i diversitats sexuals i en labordatge de les violències masclistes en joves } \\
\text { adreçades a professionals de la joventut. }\end{array}$ \\
\hline $\begin{array}{l}\text { Plan para la igualdad } \\
\text { y contra la violencia } \\
\text { de género (2013-2018) } \\
\text { CASTILLA Y LEÓN }\end{array}$ & $\begin{array}{l}\text { Acciones de sensibilización, especialmente dirigidas los hombres, para avanzar } \\
\text { en la corresponsabilidad en todos los ámbitos de la vida } \\
\text { Propiciar encuentros entre asociaciones de mujeres y asociaciones de hombres } \\
\text { pro-igualdad para facilitar el alcance de objetivos comunes } \\
\text { Desarrollar programas de intervención psicológica para agresores }\end{array}$ \\
\hline $\begin{array}{l}\text { II Plan estratégico } \\
\text { (2019-2024) } \\
\text { CASTILLA Y LA } \\
\text { MANCHA }\end{array}$ & $\begin{array}{l}\text { En la presentación se dice como una novedad del Plan ("nuevas } \\
\text { masculinidades"). Habla de "modelo masculino hegemónico". Este plan aborda } \\
\text { nuevos ámbitos como: masculinidades igualitarias o "nuevas masculinidades", } \\
\text { que cuestionan la masculinidad hegemónica y los privilegios que el sistema } \\
\text { patriarcal atribuye a los hombres. }\end{array}$ \\
\hline $\begin{array}{l}\text { III Plan de igualdad } \\
(2003-2006)^{3} \\
\text { CANTABRIA }\end{array}$ & $\begin{array}{l}\text { CONCILIACION } \\
\text { Realizar acciones de sensibilización para incentivar que los permisos y } \\
\text { licencias por razones familiares sean disfrutados por hombres. }\end{array}$ \\
\hline $\begin{array}{l}\text { Estrategia para la } \\
\text { igualdad }(2013-2020) \\
\text { CANARIAS }\end{array}$ & $\begin{array}{l}\text { Diagnóstico: los hombres presentan enfermedades más graves y fatales } \\
\text { (consecuencia, entre otros factores, de su falta de cultura preventiva y de } \\
\text { hábitos y conductas de riesgo) pero no diseña medidas para intervenir en esta } \\
\text { situación. }\end{array}$ \\
\hline III Plan estratégico & $\begin{array}{l}\text { ATENCIÓN Y PREVENCIÓN VIOLENCIA DE GÉNERO } \\
\text { Integra medidas dirigidas a sensibilizar a toda la sociedad, especialmente, a los } \\
\text { hombres }\end{array}$ \\
\hline $\begin{array}{l}\text { V Plan estratégico } \\
(2017-2021) \\
\text { EXTREMADURA }\end{array}$ & $\begin{array}{l}\text { Proyectos de capacitación en nuevas masculinidades } \\
\text { Proyectos de cooperación entre movimientos de hombres }\end{array}$ \\
\hline $\begin{array}{l}\text { VII Plan Estratégico } \\
(2017-2020) \\
\text { GALICIA }\end{array}$ & $\begin{array}{l}\text { Fomento del uso de permisos y medidas de conciliación que fomenten la } \\
\text { corresponsabilidad por parte de los hombres } \\
\text { Impulso de los programas de atención psicológica dirigidos a hombres con } \\
\text { problemas de control de la violencia }\end{array}$ \\
\hline
\end{tabular}




\begin{tabular}{|c|c|}
\hline PLAN & MEDIDAS \\
\hline $\begin{array}{l}\text { I Plan estratégico } \\
(2019-2022) \\
\text { LA RIOJA }\end{array}$ & $\begin{array}{l}\text { Facilitar la puesta en marcha de programas dirigidos a hombres para promover } \\
\text { su concienciación, participación e implicación en pro de la igualdad y la } \\
\text { corresponsabilidad } \\
\text { Impulsar programas de sensibilización y formación orientadas a prevenir los } \\
\text { riesgos de salud en los chicos y hombres derivados de su socialización de género } \\
\text { VIOLENCIA } \\
\text { Impulsar la creación de asociaciones o grupos de reflexión de hombres en } \\
\text { relación con las nuevas masculinidades }\end{array}$ \\
\hline $\begin{array}{l}\text { Estrategia } \\
\text { para la Igualdad } \\
(2018-2021) \\
\text { MADRID }\end{array}$ & $\begin{array}{l}\text { Concienciar de la importancia de la educación por las familias en la igualdad } \\
\text { entre géneros y de la implicación del hombre en la conciliación, sensibilizando } \\
\text { sobre la sobrecarga de trabajo realizado por las mujeres } \\
\text { Proponer al Gobierno la adopción de medidas de mejora de los permisos y } \\
\text { licencias vinculados a la paternidad y a la maternidad }\end{array}$ \\
\hline $\begin{array}{l}\text { II Plan estratégico } \\
(2018-2022) \\
\text { MEILLA }\end{array}$ & $\begin{array}{l}\text { JUVENTUD Y EDUCACIÓN EN VALORES } \\
\text { Los hombres deben estar igual de implicados que las mujeres, asumiendo su } \\
\text { corresponsabilidad } \\
\text { CONCILIACIÓN Y CORRESPONSABILIDAD } \\
\text { Diagnóstico: "no ha llevado consigo la involucración de los hombres al trabajo } \\
\text { doméstico, pues, en la mayoría de las familias, las tareas domésticas siguen } \\
\text { recayendo sobre las mujeres" }\end{array}$ \\
\hline $\begin{array}{l}\text { V Plan estratégico } \\
\text { (2016-2020) MURCIA }\end{array}$ & $\begin{array}{l}\text { VIOLENCIA. Desarrollo de acciones que contribuyan a evitar } \\
\text { comportamientos de pareja tóxicos y violentos, trabajando tanto con mujeres } \\
\text { como con hombres } \\
\text { Talleres prevención de violencia de género en población reclusa }\end{array}$ \\
\hline $\begin{array}{l}\text { I Plan de igualdad } \\
(2006-2010) \\
\text { NAVARRA }^{4}\end{array}$ & $\begin{array}{l}\text { SALUD Y BIENESTAR. Impulsar la presencia y participación activa de } \\
\text { los padres en los cursos, y programas prenatales de preparación al parto } \\
\text { y cuidados con el fin de implicarles en una paternidad más activa y } \\
\text { corresponsable } \\
\text { CONCILIACIÓN. Incentivar la utilización, por parte de los hombres, de } \\
\text { permisos y licencias para el cuidado de personas dependientes. }\end{array}$ \\
\hline $\begin{array}{l}\text { VII Plan para la } \\
\text { igualdad } \\
\text { PAIS VASCO }\end{array}$ & $\begin{array}{l}\text { EMPODERAMIENTO MUJERES. Indicador: normalizar la participación de } \\
\text { los chicos en ámbitos o actividades no masculinizadas } \\
\text { TRANFORMACIÓN DE LA ECONOMÍA. Aumentar el número de } \\
\text { hombres jóvenes y adultos que cuestionan el modelo tradicional masculino } \\
\text { y desarrollan actitudes y comportamientos coherentes con la igualdad y } \\
\text { comprometidos con los trabajos de cuidados. } \\
\text { Incrementar el número de hombres que se acogen a medidas para la } \\
\text { conciliación corresponsable. Indicador: Incremento del porcentaje de } \\
\text { matriculaciones de hombres en las titulaciones de formación profesional de } \\
\text { grado medio y superior, y en los grados universitarios que cuentan con más de } \\
\text { un } 75 \% \text { de matriculación femenina. } \\
\text { Aumentar el número de hombres que cuestionan el modelo tradicional } \\
\text { masculino y desarrollan actitudes y comportamientos coherentes con la } \\
\text { igualdad y comprometidos con los trabajos de cuidados. Indicador: Evolución } \\
\text { de la participación de los hombres en acciones de formación y sensibilización } \\
\text { en igualdad, en el marco de Gizonduz. } \\
\text { VIOLENCIA. Incrementar el número de chicos y hombres, que participan en } \\
\text { programas y actividades que visibilicen la relación entre la desigualdad y la } \\
\text { violencia contra las mujeres o que procuren su prevención }\end{array}$ \\
\hline
\end{tabular}




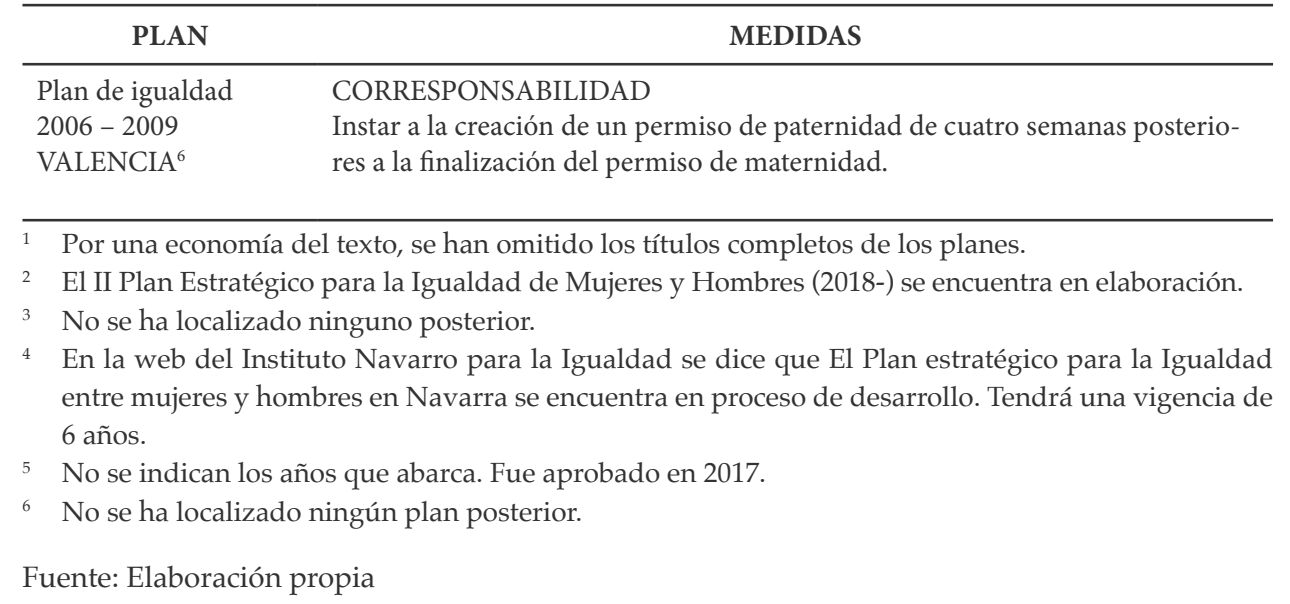

Bakea Alonso Fernández de Avilés. Licenciada en Sociología por la Universidad de Salamanca, Master en Problemas Sociales (UNED), Título de Experta en Políticas Europeas de Género por la UCM y suficiencia investigadora en el Programa de Doctorado "La Perspectiva feminista como Teoría Crítica" (UCM). Autora de guías y materiales en las temáticas de violencia de género, masculinidades y migraciones. Actualmente es Doctoranda en Trabajo Social en la Universidad Complutense de Madrid. Forma parte del equipo de coordinación del Area de Igualdad de la Fundación Cepaim.

Correo electrónico: bakea@cepaim.org

Artículo recibido el 20 de Enero y aceptado para su publicación el 05 de Marzo de 2021. 